\title{
La influencia de la cultura organizacional y la capacidad de absorción sobre la transferencia de conocimiento tácito intra-organizacional
}

\author{
AURORA IRMA MÁYNEZ-GUADERRAMA, Ph.D. ${ }^{1,2}$ \\ Profesora-Investigadora, Universidad Autónoma de Ciudad Juárez, México. \\ auroramaynez@yahoo.com
}

JUDITH CAVAZOS-ARROYO, Ph.D.

Profesora-Investigadora, Universidad Popular Autónoma del Estado de Puebla, México. cavazosjudith01@gmail.com

\begin{abstract}
JOSÉ PABLO NUÑO-DE LA PARRA, Ph.D.
Director de Relaciones Internacionales de Posgrado e Investigación del Sistema, Universidad Popular Autónoma del Estado de Puebla, México.

pablo.nuno@upaep.mx
\end{abstract}

\section{RESUMEN}

En este artículo se analiza la influencia de la capacidad de absorción y la cultura organizacional como factores requeridos para que la transferencia de conocimiento sea exitosa y eficiente. La investigación fue de naturaleza cuantitativa, empírica y de sección transversal. La información se examinó, en una primera etapa, a través de un Análisis Factorial Confirmatorio (AFC), y posteriormente mediante un Sistema de Ecuaciones Estructurales basado en Covarianzas (CBSEM). Los resultados indican que ambas variables inciden positiva y significativamente sobre la transferencia de conocimiento intra-organizacional, y que en el caso de la cultura, existe una influencia directa y otra indirecta, mediada por la capacidad de absorción.

Palabras clave. Transferencia de conocimiento; cultura organizacional; capacidad de absorción.

Recibido: 14-jun-11, corregido: 27-abr-12 y aceptado: 26-jun-12

Clasificación JEL: L29; M10; M14

@

\footnotetext{
1 Este documento fue seleccionado en la convocatoria para enviar artículos, Call for Papers, realizada en el marco del “II Simposio Iberoamericano de Estudios Gerenciales: Una mirada interdisciplinar a la innovación”, organizado por la revista académica Estudios Gerenciales bajo la dirección de la Facultad de Ciencias Administrativas y Económicas de la Universidad Icesi; el evento tuvo lugar los días 12, 13 y 14 de octubre de 2011, en la ciudad de Cali (Colombia). Este documento fue presentado en las sesiones simultáneas del área de "Innovación en transferencia del conocimiento". 2 Autor para correspondencia. Dirigir a: Palma Areca 4750-6, Fracc. Paseo de las Palmas, Ciudad Juárez, Chih., México C.P. 32664, México.
} 
La influencia de la cultura organizacional y la capacidad de absorción sobre la transferencia de conocimiento tácito intra-organizacional

\section{The influence of organizational culture and absorptive capacity on the transfer of tacit intra-organizational knowledge}

This article provides a review of the impact of absorptive capacity and organizational as key determining factors of successful and efficient knowledge transfer. This research work is quantitative, empirical, and cross sectional in nature. The information was first examined at an early stage through a confirmatory factorial analysis (CFA) and then using a covariance-based structural equation system (СBSEM). The results indicate that both variables have a positive and significant effect on intra-organizational knowledge transfer and that, in the case of culture, there is both a direct and indirect influence that is mediated by absorptive capacity.

Keywords. Knowledge transference; corporate culture; absorptive capacity.

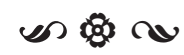

\section{A influência da cultura organizacional e a capacidade de absorção sobre a transferência de conhecimento tácito intraorganizacional}

Este artigo analisa a influência da capacidade de absorção e da cultura organizacional como fatores necessários para que a transferência de conhecimentos seja bem sucedida e eficiente. A pesquisa foi de natureza quantitativa, empírica e de seção transversal. A informação foi examinada, em uma primeira etapa, através de uma Análise Fatorial Confirmatória (AFC), e posteriormente, através de um sistema de Equações Estruturais baseado em Covariâncias (CBSEM). Os resultados indicam que ambas as variáveis incidem positiva e significativamente sobre a transferência de conhecimento intraorganizacional, e que no caso da cultura, existe uma influência indireta e outra direta, mediada pela capacidade de absorção.

Palavras-chave. Transferência de conhecimento; cultura organizacional; capacidade de absorção. 


\section{Introducción}

Los cambios y presiones de una economía global, rápidamente cambiante y basada en la información, han provocado que el conocimiento se reconozca como un activo vital para las organizaciones (Herschel \& Nemati, 2000). El desempeño organizacional se explica a partir de lo que una organización sabe, es decir, se basa en su conocimiento (Argote \& Ingram, 200o). Este activo intangible faculta a los individuos y a las empresas en materia de resolución de problemas y competitividad (Evanschitzky, Ahlert, Blaich \& Kenning, 2007), y se ubica precisamente en el núcleo de los procesos de innovación (Simonen \& McCann, 2010). Las empresas en industrias rápidamente cambiantes, derivan sus ventajas competitivas primarias a través de la habilidad de sus empleados de crear y gestionar el conocimiento (Collins \& Smith, 2006) y, sin importar el tipo de organización, la necesidad para transferir de forma efectiva el conocimiento es importante (Collins \& Hitt, 2006).

La transferencia de conocimiento intraorganizacional estimula la creación de nuevo conocimiento, incrementa las habilidades de la firma para innovar (Tsai, 2001) y, en consecuencia, incrementa el nivel de innovación y se relaciona positivamente con la ventaja competitiva (Wijk, Jansen \& Lyles, 2008). Para que las prácticas de compartir conocimiento sean efectivas, resulta vital la comprensión de los factores organizacionales que las afectan (Hsu, 2008).

En trabajos empíricos desarrollados en lo referente a la transferencia del conocimiento, los académicos (Herschel \& Nemati, 2000; Hsu, 2008; Riege, 2005; Szulanski, 1996; Wong \& Aspinwall, 2005) han estudiado diversos factores de tipo conceptual, organizacional, personal, o tecnológico, así como las diferentes combinaciones entre éstos. Para algunos (Lucas \& Ogilvie, 2006), la transferencia puede comprenderse mejor si en el análisis se incluye la cultura en la que ocurren las interacciones. Otros trabajos (Herschel \& Nemati, 2000; Wong \& Aspinwall, 2005) la señalan como elemento crítico en el éxito de la misma.

Por otra parte, uno de los constructos más prominentes en la transferencia de conocimiento organizacional y que la impacta positivamente, es la capacidad de absorción (Lucas \& Ogilvie, 2006; Wijk et al., 2008). Además, la literatura indica que la cultura corporativa, a partir de sus valores, se relaciona con la capacidad de absorción (Harrington \& Guimaraes, 2005).

No obstante la importancia de las variables anteriores, la literatura indica la necesidad de estudiar la relación entre cultura, gestión del conocimiento e innovación, sugiriendo diseñar y probar modelos más complejos con técnicas multivariadas, como por ejemplo, introducir relaciones de mediación y de ecuaciones simultáneas utilizando modelos SEM (Donate \& Guadamillas, 2010). Además, también se indica que la mayor parte de los estudios sobre capacidad de absorción han sido a nivel conceptual, existiendo escasos estudios empíricos (Knoppen, Sáenz \& Johnston, 2011). En lo referente a la influencia de la cultura sobre la capacidad de absorción, estudios recientes (Murovec \& Prodan, 2009) indican la carencia de evidencia empírica de las relaciones entre algunas de las dimensiones de ambos constructos, señalando que solo se han estudiado de forma exploratoria (Khoja \& Maranville, 2010).

Con el objetivo de incrementar el conocimiento relativo, se diseñó y llevó a cabo un trabajo de investigación de naturaleza cuantitativa con un diseño transversal, el cual tuvo por objetivos analizar en la realidad 
empírica, bajo un enfoque confirmatorio: a) El efecto de la cultura organizacional y la capacidad de absorción sobre la transferencia de conocimiento y b) La incidencia de la cultura organizacional sobre la capacidad de absorción.

Este artículo se divide en cuatro apartados. En el primero de ellos se muestran antecedentes académicos de las variables cultura corporativa, capacidad de absorción ,y la relación de éstas con la transferencia de conocimiento tácito dentro de las organizaciones. En el segundo apartado se describe la metodología utilizada. En el tercero, se exponen los resultados describiéndose brevemente el perfil de los sujetos participantes y de las organizaciones en que laboran; asimismo se expone el modelo conceptual contrastado en la realidad empírica y la verificación de las hipótesis planteadas. Finalmente, se presentan la discusión y conclusiones del trabajo realizado.

\section{Revisión de literatura}

La cultura de la empresa es una estructura invisible lo suficientemente poderosa para definir las normas y reglas que los empleados deberán seguir, a la vez que éstas determinan el desempeño de la organización (Itami \& Roehl, 1987). A través de normas compartidas por los miembros del grupo, la cultura corporativa guía y restringe los comportamientos de éstos (Schein, 2004). Esta variable parte del grupo de valores y suposiciones que afectan en su evolución a otros de sus elementos, entre los que se encuentran los símbolos, rituales, normas y actividades (Kostova, 1999).

De forma típica, los investigadores coinciden en que la cultura empresarial puede definirse como el agregado de cuestiones cognitivas compartido por los miembros de una unidad social (O'Reilly, Chatman \& Caldwell, 1991). En este, se agregan las creen- cias, valores, actitudes y hábitos permeados entre los grupos e individuos que conforman la organización (Hall, 1992). De acuerdo a Schein (2004), la cultura empresarial es el patrón de supuestos básicos compartidos aprendido por el grupo conforme soluciona sus problemas de unificación interna y de adaptación externa, siendo éste el resultado de un adecuado funcionamiento, ya que se considera válido y se les enseña a los nuevos miembros como la forma adecuada de percibir, sentir y pensar con respecto a ciertos problemas.

La cultura corporativa forma parte del ADN grupal y el éxito en los procesos cuya meta sea aprender, requiere de la preexistencia de ciertos genes organizacionales (Schein, 2004). La existencia de ideas ligadas a los valores culturales de la organización, podrían afectar la forma en que las personas comparten su conocimiento dentro de la misma (Yoo \& Torrey, 2002). Dado que refleja los valores y normas de una organización, esta variable juega un papel importante en la delimitación de las áreas en las cuales la organización será capaz de aprender, así como de aquellas en las que se mostrará reacia a modificar sus políticas (Harrington \& Guimaraes, 2005). El sistema de valores corporativos determina los tipos deseados de conocimiento (así como las clases de actividades de gestión del conocimiento relacionadas), que se consideran adecuadas y por tanto son tolerados y alentados (Gold, Malhotra \& Segars, 2001).

No obstante, al no existir mediciones claras, visibles, universales ni genéricas de la transferencia de conocimiento, para su estudio es necesario elegir los indicadores convenientes, debiendo discernirse entre un grupo integrado de acciones, comportamientos, actitudes y resultados diseñados para ambientes culturales específicos (Perrin, Rolland \& Stanley, 2007). En esa 
vertiente, Cameron \& Quinn (2006), basándose en el modelo teórico conocido como Competing Values Framework, plantearon un marco referencial que incluye cuatro tipos de cultura dominante: jerárquica, de mercado, de clan y adhocrática.

En este trabajo de investigación se toman como referencia dos de los tipos de cultura planteados por dicho modelo: las organizaciones tipo clan y las adhocráticas. En el caso de las primeras, se afirma que son entidades permeadas por valores y metas compartidos, donde se fomenta la cohesión, la participación, la individualidad y el sentido grupal, lo cual conlleva al trabajo en equipo y al desarrollo de los empleados, a través de la provisión de medios y recursos que faciliten su participación y compromiso. En el caso de las segundas, se indica que en ellas se fomenta la adaptabilidad, la flexibilidad y la creatividad, y que se enfrentan al reto de producir bienes o servicios innovadores, adaptándose rápidamente a las nuevas oportunidades.

1.1 La cultura organizacional y la transferencia de conocimiento tácito dentro de las organizaciones

El conocimiento y la cultura están indisolublemente vinculados en las organizaciones (De Long \& Fahey, 2000). La cultura crea el contexto para la interacción social, y por tanto, influye sobre la gestión efectiva y exitosa del conocimiento organizacional (De Long \& Fahey, 2000; Donate \& Guadamillas, 2010; Yoo \& Torrey, 2002). Con respecto a la transferencia de conocimiento, se afirma que la cultura ejerce una influencia positiva y significativa (Lucas \& Ogilvie, 2006).

En los estudios previos, el tema subyacente ha sido que determinados tipos de valores organizacionales derivan en ciertas clases de comportamientos de gestión del conocimiento y que tales comportamientos, pueden provocar ciertos resultados. Estos resultados pueden ser los valores culturales, como compartir, la confianza y la apertura, los cuales incidirán positivamente sobre los comportamientos de la gestión del conocimiento, por ejemplo, para contribuir y compartir (Alavi, Kayworth \& Leidner, 2005). Luego, las empresas con orientaciones de valor más abierto y de apoyo, estarán predispuestas a comportamientos de conocimiento constructivo, como por ejemplo, el que los miembros de la firma compartan con otros lo que saben (Gold et al., 2001).

La cultura corporativa proporciona a cada empleado un método común y distintivo para transmitir y procesar información (Itami \& Roehl, 1987). Los valores culturales se consideran significativos para facilitar la transferencia de conocimiento, sobre todo la del conocimiento tácito (Donate \& Guadamillas, 2010). Por ello, se reconoce la cultura como un aspecto relevante, tanto en términos del conocimiento tácito como del método para compartirlo (Jonsson \& Kalling, 2007). Luego, es razonable pensar que la disposición y promoción de las prácticas de transferencia de conocimiento dentro de la empresa derivarán en mayores resultados de ésta (Donate \& Guadamillas, 2010).

Los valores subyacentes en los tipos de cultura clan/adhocrática soportan, entre otras actividades, la participación, el trabajo de equipo, el desarrollo de empleados y la producción de bienes y servicios innovadores, proveyendo de medios y recursos que faciliten la participación y el compromiso. En consecuencia, y con base en los argumentos expuestos, se propone la siguiente hipótesis de investigación:

Hipótesis 1 (H1). La cultura organizacional tipo clan/adhocrática, incide positivamente en la transferencia de conocimiento tácito dentro de las organizaciones. 


\subsection{Capacidad de absorción}

Las empresas deben motivar a su personal para que aprenda y absorba nuevo conocimiento, ya que existe la posibilidad de indisposiciones por factores como la comodidad que brinda el status quo, el miedo a la incertidumbre, la preocupación por la posibilidad de perder privilegios existentes, y/o el desinterés de buscar o compartir conocimiento (Zhao \& Anand, 2009). La falta de motivación, la ausencia de capacidad de absorción y la insuficiente capacidad de retención, son barreras receptivas citadas frecuentemente (Lin, Tan \& Chang, 2008).

Uno de los constructos más prominentes en la literatura de transferencia de conocimiento organizacional es la capacidad de absorción (Wijk et al., 2008), y conjuntamente con el aprendizaje, son factores mencionados asiduamente como elementos explicativos de dicha actividad (Reagans \& McEvily, 2003). No obstante lo anterior, los antecedentes sobre dicha variable han sido fuertemente ignorados (Wijk et al., 2008).

La capacidad de absorción se define típicamente como la habilidad de una organización para reconocer información nueva y valiosa, asimilarla con el conocimiento existente y aplicarla en fines comerciales y/o en la creación de nuevas capacidades (Cohen \& Levinthal, 1990; Collins \& Hitt, 2006; Szulanski, 1996; Wijk et al., 2008; Zhao \& Anand, 2009). Cimentar una capacidad de absorción implica construir estructuras traslapadas de conocimientos compartidos dentro de la organización (Harrington \& Guimaraes, 2005), por lo que dicha variable es relativa y dependiente de la combinación específica del emisor-receptor de que se trate (Lane \& Lubatkin, 1998). Este constructo alude a la capacidad del aprendiz para absorber y explotar nuevas ideas o conocimientos, precisando que debe contarse con la habili- dad de relacionar conceptualmente la causa y el efecto (Peansupap \& Walker, 2009).

Tanto a nivel individual como organizacional, la absorción de conocimiento depende de la capacidad del receptor para añadir nuevos conocimientos a los ya existentes (Grant, 1996). Los individuos pueden encontrar algunos tipos de conocimiento más difíciles de absorber que otros, debido a las características específicas del conocimiento (Tallman, Jenkins, Henry \& Pinch, 2004), sea por su carácter tácito (Reed \& De Fillippi, 1990), su complejidad o contexto (Tsoukas \& Vladimirou, 2001). Existen varias barreras vinculadas al conocimiento transmitido (Minbaeva, 2007; Szulanski, 1996), fuente de conocimiento (baja confiabilidad, falta de motivación y capacidad de diseminación), del receptor de los conocimientos (falta de motivación, capacidad de absorción y capacidad de retención) o del contexto (estéril, organizacional y de relaciones arduas).

La capacidad de absorción tiene dos elementos importantes, la base de conocimiento existente y la intensidad del esfuerzo (Kim, 1999), y cuenta con cuatro dimensiones complementarias: adquisición, asimilación, transformación y explotación de conocimiento (Simonin, 2004; Zahra \& George, 2002). Es un constructo multidimensional que involucra la habilidad para adquirir, asimilar, transformar y aplicar el conocimiento, y puede entenderse también como la combinación del esfuerzo y la base previa de dicho activo intangible (Zahra \& George, 2002).

Con respecto al primer elemento de este constructo, investigaciones anteriores indican que únicamente aquellos con un nivel previo de conocimiento compartido tienen la capacidad de intercambiarlo (Liyanage, Elhag, Ballal \& Li, 2009). Una de las formas más importantes para el aprendizaje de nuevos conceptos es la asociación de éstos con lo 
ya conocido: para las personas es más fácil absorber nuevos conceptos en áreas en las que tienen cierta experiencia, y les resulta más complicado adquirirlos fuera de su experiencia inmediata (Reagans \& McEvily, 2003). Las bases previas permiten asimilar, encontrar sentido, utilizar y explotar el nuevo conocimiento (Cohen \& Levinthal, 1990; Inkpen \& Pien, 2006; Kim, 1999). Los teóricos de la organización visualizan la falta de conocimiento relacionado como la barrera más significativa para transferir este activo intelectual en las empresas (Dixon, 2002).

Con respecto al segundo elemento, la literatura (Cohen \& Levinthal, 1990; Kim, 1999) indica que, para desarrollar una efectiva capacidad de absorción, se requiere esforzarse de forma intensa, ya que resulta insuficiente sólo exponer a las personas con el conocimiento relevante, omitiendo ejercer el esfuerzo necesario para que éste se internalice. La intensidad del esfuerzo o compromiso se refiere a la cantidad de energía que las personas destinan en una actividad o tarea (Kim, 1999). Además, debe resaltarse que la capacidad de utilizar el conocimiento previo depende, en gran medida, de los nuevos conocimientos que sean adquiridos (Penrose, 1962). Absorber y adaptar nuevo conocimiento implica cambios en la forma en que las personas piensan, se comportan y realizan sus funciones productivas (Zhao \& Anand, 2009).

El emisor debe ser capaz de explicar el conocimiento, el receptor debe ser capaz de integrarlo y reconstruirlo (Evanschitzky et al., 2007), y una condición previa para transferir conocimiento es que el receptor no se resista a utilizar el conocimiento nuevo -síndrome de no-inventado-aquí-, además de que cuente con suficiente conocimiento relacionado que le permita apreciar el valor del nuevo (Lin et al., 2008). Esta variable faculta a las firmas para asimilar y aplicar conocimiento (Wijk et al., 2008), y es dependiente de la capacidad individual de absorción de sus miembros (Cohen \& Levinthal, 1990).

\subsection{Capacidad de absorción y transferencia} de conocimiento tácito intra-organizacional La capacidad de absorción es una capacidad dinámica (Swart, van den Hooff \& van Baalen, 2011) que tiene un papel indispensable en incrementar la transferencia de conocimiento intra e interorganizacional cuando se busca incorporar los conocimientos compartidos en los sistemas y procesos de la organización (Wijk et al., 2008). Es de resaltar que algunos trabajos empíricos no encontraron evidencia de dicha relación (Lane \& Lubatkin, 1998), y por ello es que debe seguirse explorando ésta, considerando de forma importante el contexto.

Si el conocimiento no es absorbido por el receptor, significa que éste no se ha transferido (Davenport \& Prusak, 20oo). Deng, Doll \& Cao (2008) exploraron la capacidad de absorción como una barrera de base individual que ocurre cuando los empleados interactúan con artefactos de tecnologías de la información. Sus resultados mostraron que la falta de capacidad de absorción en el contexto de las tecnologías de la información resultaba en una pobre innovación y productividad de los ingenieros.

Por otro lado, Minbaeva (2007) estudió la transferencia de conocimiento a través de procesos cara a cara dentro de empresas multinacionales de origen danés, los resultados mostraron que una capacidad de absorción pobre, incompatibilidad cultural y altos niveles de conocimiento tácito y ambigüedad causal, resultan en ineficiencias sustanciales en la transferencia de conocimiento. Un fuerte sentimiento de propiedad personal del conocimiento afecta negativamente a la disposición de la gente 
La influencia de la cultura organizacional y la capacidad de absorción sobre la transferencia de conocimiento tácito intra-organizacional

para compartir conocimientos y entablar una comunicación abierta, culminando en la adopción de intentos competitivos y comportamientos contraproducentes entre los empleados (Hasnain \& Jasimuddin, 2012). Comparativamente con el conocimiento explícito, la capacidad de absorción tiene mayor influencia en la transferencia de conocimiento tácito (Santoro \& Bierly, 2006).

La capacidad de absorción involucra un conjunto de habilidades necesarias para tratar con el componente tácito de los conocimientos transferidos y la necesidad de modificar este conocimiento, así como la capacidad de aprender y resolver problemas (Zahra \& George, 2002). Una empresa necesita tiempo y personas para adquirir conocimientos y estas dos variables afectan la acumulación de éstos, lo que determina su capacidad de absorción para comprenderlos y utilizarlos (Chan, Pretorius \& Oerlemans, 2012).

El éxito para la asimilación de nuevos conocimientos tácitos radica en la capacidad del equipo receptor para asimilarlos, es decir, su capacidad de absorción (Tsai, 2001), ya que una vez que el nuevo conocimiento se asimila y se pone en práctica, puede favorecer el desarrollo de la innovación a través de nuevos productos o procesos (Lichtenthaler, 2009; Miller, Fern \& Cardinal, 2007; Seidlerde Alwis \& Hartmann, 2008). Compartir una base de conocimiento común, tanto técnica como organizacional, facilita la transferencia inter-grupal de conocimiento (Kogut \& Zander, 1992; Reagans \& McEvily, 2003). A partir de lo anterior puede plantearse:

Hipótesis 2 (H2). La capacidad de absorción influye positiva y significativamente en la transferencia de conocimiento tácito intraorganizacional.

\subsection{Relación entre cultura organizacional y capacidad de absorción}

Si lo que se busca es aprender respecto de los motivos subyacentes del comportamiento organizacional y/o comprender el efecto de la capacidad de absorción sobre el aprendizaje de las empresas, analizar la cultura corporativa es una actividad requerida (Khoja \& Maranville, 2010). Esta variable afecta el deseo y la reacción de una empresa ante la información que obtiene y transforma en conocimiento organizacional (Harrington \& Guimaraes, 2005). Comparativamente con culturas donde predomina la rutina, la cultura caracterizada por un espíritu innovador y de aprendizaje ofrecerá mayores capacidades organizacionales para compartir, asimilar y utilizar el conocimiento (Xinhua \& Cuiling, 2008).

A partir de sus valores, la cultura corporativa se relaciona con la capacidad de absorción (Harrington \& Guimaraes, 2005). Las organizaciones que promueven compartir e intercambiar recursos y capacidades, alientan a las distintas áreas para que afiancen su capacidad de absorción, fomentando que se maximice el conocimiento adquirido de otras fuentes (Khoja \& Maranville, 2010). Mientras que la capacidad de absorción se enfoca hacia la habilidad para implementar innovaciones, la cultura organizacional lo hace respecto a los valores y normas relativos al cambio y a la tecnología (Harrington \& Guimaraes, 2005).

No obstante que algunos estudios han mostrado el impacto de ciertos aspectos culturales, entre otros la capacidad de coordinación, la participación en toma de decisiones y la capacidad de inter-relación sobre la capacidad de absorción; en dichos trabajos no se han identificado explícitamente estos factores como cultura organizacional (Khoja \& Maranville, 2010), y en consecuencia, de acuerdo con los postulados de Murovec \& 
Prodan (2009), existe una gran carencia de evidencia empírica respecto de la influencia de la cultura sobre la capacidad de absorción. Hasta donde llega el conocimiento, solamente se han analizado de forma empírica las relaciones entre algunas de las dimensiones de la cultura organizacional y la capacidad de absorción, y dichos análisis se han realizado desde un enfoque exploratorio (Khoja \& Maranville, 2010). De acuerdo a los argumentos presentados, resulta lógico esperar que:

Hipótesis $3\left(\mathrm{H}_{3}\right)$. La cultura organizacional incide directa, positiva y significativamente sobre la capacidad de absorción.

El modelo básico que guía la investigación se presenta en el Gráfico 1.

\section{Metodología}

Para alcanzar el objetivo propuesto se diseñó una investigación cuantitativa, de naturaleza empírica y de sección transversal, basada en la revisión de la literatura realizada de las variables cultura organizacional, capacidad de absorción y transferencia de conocimiento Tácito intra-organizacional. Para contrastar las hipótesis del modelo teórico propuesto en su conjunto, se utilizó un sistema de ecuaciones estructurales basado en covarianzas, ya que dicha técnica estadística es considerada útil para investigar la plausibilidad de modelos teóricos que pudieran explicar las interrelaciones entre un grupo de variables en diversas disciplinas científicas ( $\mathrm{Hu} \&$ Bentler, 1999).

El estudio se desarrolló en empresas participantes en las industrias automotriz, cementera, médica, electrónica y de telecomunicaciones, ubicadas en las ciudades de Juárez y Chihuahua en el estado fronterizo de Chihuahua, México. La muestra fue de tipo no probabilística, la cual incluyó sujetos que laborasen como gerentes, supervisores, analistas, ingenieros y técnicos, en el contexto antes descrito. Como técnica para recolectar la información se utilizó la encuesta,

Gráfico 1. Modelo conceptual propuesto

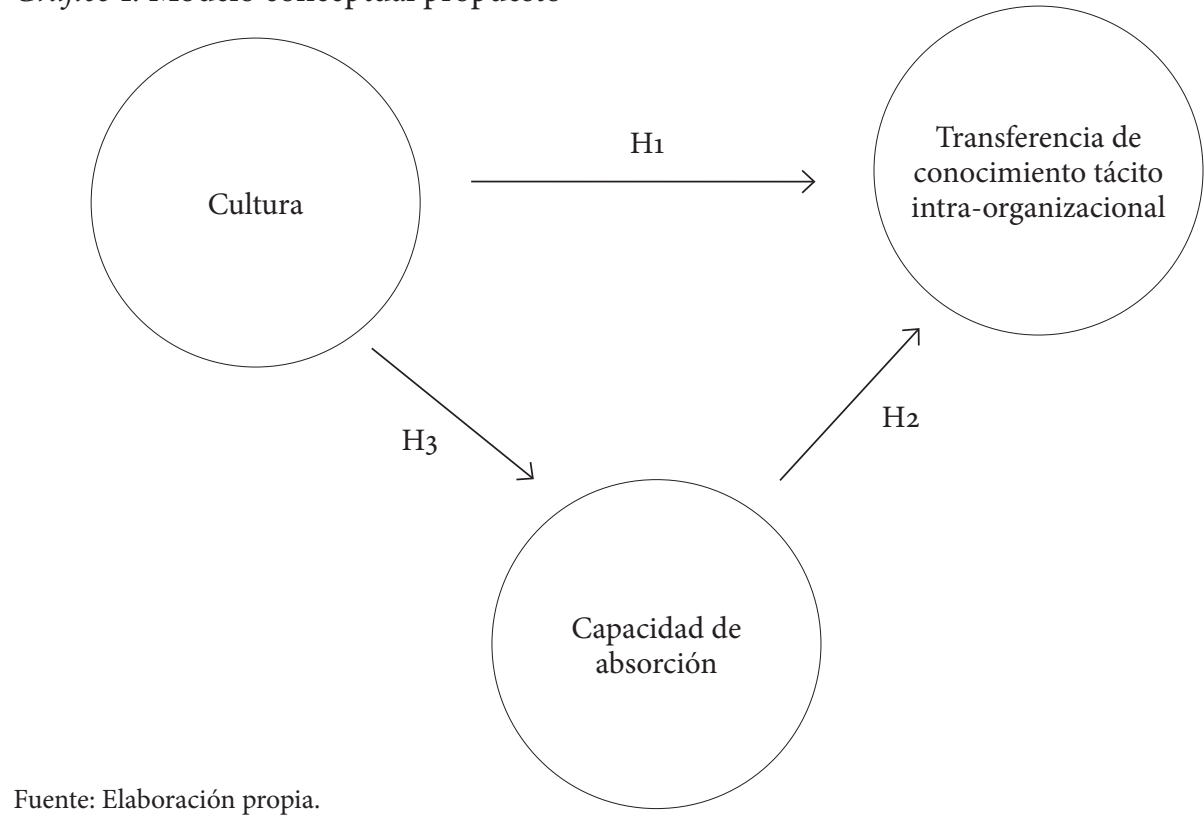


la cual se realizó a través de un cuestionario auto-administrado aplicado individualmente en el contexto laboral durante el período del 8 de Octubre al 9 de Noviembre del 2010.

En total se recibieron 340 cuestionarios, 62 de los cuales fueron desechados (49 por responder parcialmente, 11 por corresponder a localidades distintas a las delimitadas previamente, y los 2 restantes por no autorizar el uso agregado de su información). El total de instrumentos utilizables fue 278 , representando el $81,76 \%$ de los recibidos. En el cuestionario se incluyeron ítems diseñados con un formato tipo Likert (1932), con cinco puntos de asignación de respuesta, dónde 1 significó "totalmente de acuerdo" y 5 "totalmente en desacuerdo". Lo anterior permitió que los participantes seleccionaran su grado de acuerdo o desacuerdo con las afirmaciones planteadas (Hair, Bush \& Ortinau, 2007). Cabe señalar que este trabajo forma parte de un proyecto de investigación más amplio, y que además de las variables utilizadas en éste, en el instrumento aplicado fueron incluidas otras, todas enfocadas hacia la transferencia de conocimiento tácito dentro de la organización.

La operacionalización de las variables se realizó inicialmente a través de 18 ítems: cultura organizacional (5), capacidad de absorción (6) y transferencia de conocimiento (7). Para el análisis de los datos se siguió el procedimiento de dos etapas recomendado por Anderson \& Gerbing (1988). En la primera etapa, a fin de evaluar las propiedades psicométricas del modelo de medida, se llevó a cabo un Análisis Factorial Confirmatorio (AFC) de segundo orden, y en la segunda, se agregaron las relaciones estructurales propuestas entre las variables latentes, las cuales fueron analizadas mediante un Sistema de Ecuaciones Estructurales (CBSEM).

La estimación de las relaciones estructurales entre las variables estudiadas se realizó con apoyo del software EQS 6.1. Tanto el AFC como el SEM se estimaron a través del Método de Máxima Verosimilitud, utilizando la corrección estadística propuesta por Satorra \& Bentler (1994) de estadísticos robustos, ya que no fue satisfecha la condición de normalidad multivariante de los datos (Coeficiente normalizado de Mardia estimado $=62,98$ ).

Con respecto a la variable capacidad de absorción, la literatura indica que es un constructo complejo y potencialmente multidimensional, y resulta cuestionable que una medición unidimensional logre su evaluación completa; además, no obstante que varios estudios empíricos han abordado esta variable, trabajos previos señalan que no se cuenta con una medición válida que incorpore todas sus dimensiones (Flatten, Engelen, Zahra \& Brettel, 2011).

En este trabajo, la capacidad de absorción se midió a nivel individual, ya que de acuerdo a los postulados de Cohen \& Levinthal (1990), este constructo organizacional es dependiente de la capacidad que en lo individual tienen sus empleados. Por ello, se diseñaron seis ítems que cubren sus dos elementos importantes (Kim, 1999), y sus cuatro dimensiones complementarias (Simonin, 2004; Zahra \& George, 2002).

Con base en los resultados del AFC, los dos ítems que medían la intensidad del esfuerzo y la base de conocimiento existente fueron eliminados del modelo de medición. Además, de acuerdo a los elementos teóricos disponibles que afirman que se trata de un constructo potencialmente multidimensional, se decidió correr - con los cuatro ítems remanentes- un análisis factorial exploratorio (AFE) para identificar la posible existencia de factores comunes. Los resultados de dicho análisis permitieron identificar dos componentes que agrupan la capacidad del individuo para: a) ser receptivo y tener la capacidad de adquirir y asimilar conoci- 
miento, y b) darse cuenta de los beneficios y lograr transformar y explotar sus habilidades. Cabe señalar que estos componentes son coincidentes con la sub-clasificación de la variable en capacidad de absorción potencial y capacidad de absorción real propuesta por Zahra \& George (2002). A través de los mismos, fue posible explicar el $77,97 \%$ de la varianza, obteniéndose un índice $\mathrm{KMO}=0,616$, y una $\mathrm{p}=0,000$ en el test de esfericidad de Bartlett.

Con respecto a la variable cultura, como se comentó de manera previa, en esta investigación se estudian dos de los tipos propuestos por el modelo Competing Values Framework: las organizaciones tipo clan y las adhocráticas. A partir de las características de estos tipos de cultura, se elaboraron cinco ítems utilizados para su medición. Como resultado del AFC se eliminó un ítem. Los cuatro ítems remanentes miden si en la empresa se fomenta el trabajo de equipo y la discusión abierta de problemas, se da aliento a actitudes como la creatividad y la flexibilidad, y se brindan los recursos, espacios y medios necesarios para aprender y compartir.

En lo relativo a la transferencia de conocimiento intra-organizacional, se incluyeron originalmente siete ítems, de los cuales fueron eliminados tres de acuerdo a los resultados del AFC. Con los cuatro ítems restantes se busca medir: 1) la existencia de cambios en modelos mentales, bases de conocimiento, desempeño, prácticas y políticas y/o comportamientos; y 2) con base en los conocimientos transferidos, el desarrollo de nuevas ideas, prácticas y/o políticas organizacionales.

Los resultados del AFC se presentan en la Tabla 1. En términos generales, el modelo de medida tiene un buen ajuste, toda vez que los indicadores IFI $=0,928$, RMSEA $=0,074$ y CFI $=0,927$; cumplen con los criterios de corte convencionales, a excepción de $\chi^{2}$, pero con respecto a este indicador es bien conocida su sensibilidad respecto al tamaño de muestra ya que conforme éste se incrementa, las probabilidades de rechazar un modelo también aumentan - sin importar si el modelo es falso o verdadero (Bagozzi \& Yi, 1988).

En lo que a la evidencia de validez convergente se refiere, los resultados del AFC indican que todas las relaciones de los ítems con sus factores hipotéticos son significativas, que la magnitud de sus cargas estandarizadas son mayores que el valor crítico de 0,50 (Bagozzi \& Yi, 1988) y que el promedio de las cargas estandarizadas de cada factor fue superior a o,70 (Hair Jr., Anderson, Tatham \& Black, 1999).

Asimismo, en la Tabla 1 se presentan pruebas de la consistencia interna de los constructos ya que, en primer lugar, salvo el caso del constructo capacidad de absorción potencial que tiene un alfa de Cronbach de 0,665 , en el resto de los constructos ese indicador excede el valor mínimo aceptable de 0,70 (Nunnally \& Bernstein, 1994); en segundo lugar, los índices de fiabilidad compuesta (IFC) para cada uno de los factores hacen lo propio respecto al valor de referencia de o,6o (Bagozzi \& Yi, 1988), y finalmente, la mayoría de los índices de varianza extraída (AVE) de cada factor superan el valor aceptable mínimo de 0,50, haciendo notar que en el caso del constructo capacidad de absorción real, el resultado fue marginal $(0,491)$ (Fornell \& Larcker, 1981). Por tanto, es posible afirmar la existencia de validez convergente.

Con respecto a la validez discriminante, los resultados del AFC sugieren su existencia ya que ninguno de los intervalos de confian$z a$, para $\pm 2 \lambda$, comprenden la unidad y, por otro lado, ya que los valores de los AVE son ampliamente superiores a los cuadrados 
La influencia de la cultura organizacional y la capacidad de absorción sobre la transferencia de conocimiento tácito intra-organizacional

Tabla 1. Indicadores del modelo de medida

\begin{tabular}{|c|c|c|c|c|c|c|}
\hline \multirow[b]{2}{*}{ Factor } & \multirow[b]{2}{*}{ Ítem } & \multicolumn{3}{|c|}{ Fiabilidad } & \multicolumn{2}{|c|}{ Validez convergente } \\
\hline & & a & IFC & AVE & $\begin{array}{c}\lambda \\
(\text { estandarizada) }\end{array}$ & $\begin{array}{c}\lambda s \\
\text { (promedio) }\end{array}$ \\
\hline \multirow[t]{4}{*}{ Cultura } & Cult1 & 0,871 & 0,873 & 0,632 & $0,823^{* *}$ & 0,794 \\
\hline & Cult2 & & & & $0,826^{* *}$ & \\
\hline & Cult3 & & & & $0,791^{\star *}$ & \\
\hline & Cult4 & & & & $0,736^{\star *}$ & \\
\hline \multirow[t]{2}{*}{$\begin{array}{l}\text { Capacidad } \\
\text { potencial }\end{array}$} & Cap1 & 0,665 & 0,689 & 0,532 & $0,603^{* *}$ & 0,720 \\
\hline & Cap2 & & & & $0,837^{* *}$ & \\
\hline \multirow[t]{2}{*}{$\begin{array}{l}\text { Capacidad } \\
\text { real }\end{array}$} & Cap3 & 0,752 & 0,761 & 0,491 & $0,861^{\star *}$ & 0,781 \\
\hline & Cap4 & & & & $0,701^{\star *}$ & \\
\hline \multirow{4}{*}{$\begin{array}{l}\text { Transferencia } \\
\text { de cono- } \\
\text { cimiento }\end{array}$} & Transf1 & 0,846 & 0,855 & 0,599 & $0,655^{* *}$ & 0,769 \\
\hline & Transf2 & & & & $0,700^{\star *}$ & \\
\hline & Transf3 & & & & $0,879^{* *}$ & \\
\hline & Transf4 & & & & $0,840^{* *}$ & \\
\hline
\end{tabular}

\begin{tabular}{|c|c|c|c|c|c|c|}
\hline \multicolumn{5}{|c|}{ Validez discriminante } & & \\
\hline Factor & Cultura & Cap-pot & Cap-real & Transf-con & & \\
\hline Cultura & 0,632 & {$[0,317-0,007]$} & {$[0,536-0,184]$} & {$[0,605-0,341]$} & & \\
\hline Cap-pot & 0,024 & 0,532 & {$[0,636-0,284]$} & {$[0,457-0,105]$} & & \\
\hline Cap-real & 0,130 & 0,212 & 0,491 & {$[0,665-0,289]$} & & \\
\hline Transf-con & 0,224 & 0,079 & 0,228 & 0,599 & & \\
\hline $\begin{array}{l}\text { Indicadores } \\
\text { de bondad de } \\
\text { ajuste: }\end{array}$ & BBNFI & BBNNFI & CFI & IFI & MFI & RMSEA \\
\hline $\begin{array}{l}\text { S-B } \chi^{2} \quad(49 \mathrm{gl}) \\
=121,2440^{* *}\end{array}$ & 0,886 & 0,899 & 0,927 & 0,928 & 0,878 & 0,074 \\
\hline${ }^{\star *} \mathrm{P}<0,01$ & & & & & & \\
\hline
\end{tabular}

Nota. a. En el apartado de validez discriminante, en la diagonal se presentan los valores de los Ave, sobre la diagonal los intervalos de confianza y, debajo de la diagonal los cuadrados de los coeficientes de correlación entre los factores. Fuente: Elaboración propia.

de los coeficientes de correlación entre los factores. A partir de lo anterior, puede afirmarse que existe validez discriminante. En consecuencia, y de acuerdo a los elementos disponibles para la validación conjunta de los resultados, es posible confirmar la fia- bilidad, la validez convergente y la validez discriminante del modelo global de medida.

\section{Resultados}

A continuación se presenta información estadística descriptiva de los participantes 
en esta investigación, así como de las organizaciones en las que laboran. Es conveniente señalar que 7 de las 278 personas que contestaron el cuestionario, no dieron respuesta a la sección demográfica del mismo, por lo que los resultados se presentan sobre un total de 271 sujetos.

Perfil de los sujetos participantes. El 75,6\% de los participantes son hombres, el $60 \%$ indicó haber alcanzado un nivel de escolaridad de carrera profesional terminada y el $63 \%$ se encuentra en un rango de entre $26 y$ 40 años de edad (ver Tabla 2).

Perfil de las organizaciones. El 90\% de las organizaciones se localiza en Cd. Juárez, el $72,7 \%$ produce bienes para la industria Tabla 2. Perfil de los sujetos participantes ( $\mathrm{n}=271$ )

\begin{tabular}{|c|c|c|}
\hline Característica & $\begin{array}{l}\text { Número de } \\
\text { personas }\end{array}$ & $\%$ \\
\hline \multicolumn{3}{|l|}{ Sexo } \\
\hline Hombre & 205 & 75,60 \\
\hline Mujer & 66 & 24,40 \\
\hline \multicolumn{3}{|l|}{ Escolaridad } \\
\hline Preparatoria terminada & 19 & 7,00 \\
\hline $\begin{array}{l}\text { Carrera profesional } \\
\text { no terminada }\end{array}$ & 44 & 16,20 \\
\hline $\begin{array}{l}\text { Carrera profesional } \\
\text { terminada }\end{array}$ & 163 & 60,10 \\
\hline Maestría & 40 & 14,80 \\
\hline Doctorado & 1 & 0,40 \\
\hline Otra & 4 & 1,50 \\
\hline \multicolumn{3}{|l|}{ Edad } \\
\hline Menos de 21 años & 3 & 1,10 \\
\hline Entre 21 y 25 años & 32 & 11,80 \\
\hline Entre 26 y 30 años & 56 & 20,70 \\
\hline Entre 31 y 35 años & 53 & 19,60 \\
\hline Entre 36 y 40 años & 61 & 22,50 \\
\hline Entre 41 y 45 años & 35 & 12,90 \\
\hline Entre 46 y 50 años & 20 & 7,40 \\
\hline Entre 51 y 55 años & 6 & 2,20 \\
\hline Más de 56 años & 5 & 1,80 \\
\hline
\end{tabular}

Fuente: Elaboración propia. automotriz, y en el 94\% de los casos se trata de organizaciones grandes, ya que emplean a más de 250 personas (ver Tabla 3 ).

Modelo estructural. Una vez establecida la fiabilidad y validez del modelo de medida, se procedió a realizar la contrastación de las hipótesis a través del SEM correspondiente, empleando también estadísticos robustos (Satorra \& Bentler, 1994). Los resultados se presentan en la Tabla 4.

Como puede apreciarse, los estadísticos de bondad de ajuste del modelo estructural muestran también un buen ajuste global: В BNFI $=0,885 ;$ B BNNFI $=0,901 ;$ CFI $=0,927$; IFI $=0,928$; RMSEA $=0,073$; $y$ las tres hipótesis planteadas resultaron significativas para un Tabla 3. Perfil de las organizaciones $(n=271)$

\begin{tabular}{lcc}
\hline Característica & $\begin{array}{c}\text { Número de } \\
\text { personas }\end{array}$ & $\%$ \\
\hline Tamaño & & \\
$\begin{array}{l}\text { Menos de diez } \\
\text { personas }\end{array}$ & 2 & 0,70 \\
Entre diez y 30 personas & 3 & 1,10 \\
$\begin{array}{l}\text { Entre 31 y 50 } \\
\text { personas }\end{array}$ & 1 & 0,40 \\
Entre 51 y 100 & & \\
personas & 4 & 1,50 \\
Entre 101 y 250 personas & 6 & 2,20 \\
Más de 250 personas & 255 & 94,10 \\
Localización & & \\
Ciudad Juárez, Chih. & 245 & 90,40 \\
Chihuahua, Chih. & 26 & 9,60 \\
La empresa produce & & \\
bienes o servicios para la \\
industria:
\end{tabular}

Fuente: Elaboración propia. 
La influencia de la cultura organizacional y la capacidad de absorción sobre la transferencia de conocimiento tácito intra-organizacional

Tabla 4. Resultados del SEM para la contrastación de hipótesis

\begin{tabular}{|c|c|c|c|c|c|c|c|c|}
\hline Hipótesis & \multicolumn{4}{|c|}{ Relación estructural propuesta } & \multicolumn{2}{|c|}{$\begin{array}{l}\lambda(\text { estan- } \\
\text { darizada) }\end{array}$} & \multicolumn{2}{|c|}{ Resultado } \\
\hline $\mathrm{H} 1$ & $\begin{array}{l}\text { Cultura orga- } \\
\text { nizacional }\end{array}$ & $\rightarrow$ & \multicolumn{2}{|c|}{$\begin{array}{c}\text { Transferencia de conocimiento } \\
\text { tácito intra-organizacional }\end{array}$} & \multicolumn{2}{|c|}{$0,317^{\star *}$} & \multicolumn{2}{|c|}{ No rechazada } \\
\hline $\mathrm{H} 2$ & $\begin{array}{l}\text { Capacidad de } \\
\text { absorción }\end{array}$ & $\rightarrow$ & \multicolumn{2}{|c|}{$\begin{array}{c}\text { Transferencia de conocimiento } \\
\text { tácito intra-organizacional }\end{array}$} & \multicolumn{2}{|c|}{$0,403^{* *}$} & \multicolumn{2}{|c|}{ No rechazada } \\
\hline $\mathrm{H} 3$ & $\begin{array}{l}\text { Cultura orga- } \\
\text { nizacional }\end{array}$ & $\rightarrow$ & \multicolumn{2}{|c|}{ Capacidad de absorción } & \multicolumn{2}{|c|}{$0,385^{\star *}$} & \multicolumn{2}{|c|}{ No rechazada } \\
\hline \multicolumn{3}{|c|}{ Indicadores de bondad de ajuste: } & BBNFI & BBNNFI & CFI & IFI & MFI & RMSEA \\
\hline \multicolumn{2}{|c|}{$S-B \chi^{2}(49 \mathrm{gl})=121,2440^{* *}$} & & 0,885 & 0,901 & 0,927 & 0,928 & 0,878 & 0,073 \\
\hline${ }^{* *} \mathrm{p}<0,01$ & & & & & & & & \\
\hline
\end{tabular}

Fuente: Elaboración propia.

nivel de $\mathrm{p}<0,01$. En consecuencia, no es posible rechazar ninguna de ellas. En el Gráfico 2 se presenta el modelo final contrastado incluyendo los ítems de cada factor y las cargas estandarizadas. Asimismo, se muestran los diferentes indicadores estandarizados del modelo contrastado.

Una vez realizada la contrastación del modelo y antes de discutir los resultados, se consideró conveniente presentar el cálculo de los efectos totales de la cultura organizacional sobre las variables consecuentes consideradas en este trabajo, es decir, la sumatoria de sus efectos directos más sus efectos indirectos mediados por las variables correspondientes (ver Tabla 5).

En primer lugar, tal y como muestra la Tabla 5, la cultura organizacional influye directa, positiva y significativamente tanto en la transferencia de conocimiento tácito intra-organizacional $(\lambda=0,317 ; \mathrm{p}<0,01, \mathrm{H} 1$ no rechazada) como en la capacidad de absorción $(\lambda=0,385 ; \mathrm{p}<\mathrm{O}, \mathrm{O} 1$; H2 no rechazada), pero además, tal como se aprecia en la Tabla 3, la capacidad de absorción media la influencia de la cultura organizacional sobre la transferencia de conocimiento intra-organizacional $(\lambda=0,403 ; \mathrm{p}<\mathrm{O}, 01, \mathrm{H} 3$ no rechazada), es decir, existe un efecto indirecto de la cultura organizacional sobre la transferencia de conocimiento tácito intra-organizacional $(\lambda=0,155 ; \mathrm{p}<0,01)$, por lo que la influencia total ejercida por la cultura organizacional sobre la transferencia de conocimiento tácito intra-organizacional es de una magnitud considerable $\left(\lambda_{\text {total RSC-VPe }}\right.$ $=0,472 ; \mathrm{p}<0,01)$.

\section{Discusión y conclusiones}

Los resultados de esta investigación muestran que la capacidad de absorción y la cultura organizacional inciden positiva y significativamente sobre la transferencia de conocimiento tácito dentro de las organizaciones, además de que evidencian que la cultura incide también sobre la capacidad de absorción, por lo que es posible reconocer a ésta última como variable mediadora entre la cultura y la transferencia de conocimiento tácito interna.

La evidencia empírica muestra que la cultura organizacional incide directa e indirectamente sobre la transferencia de conocimiento tácito intra-organizacional. Igualmente, es de resaltar que a partir de estos resultados, puede esperarse que la 
Gráfico 2. Modelo contrastado

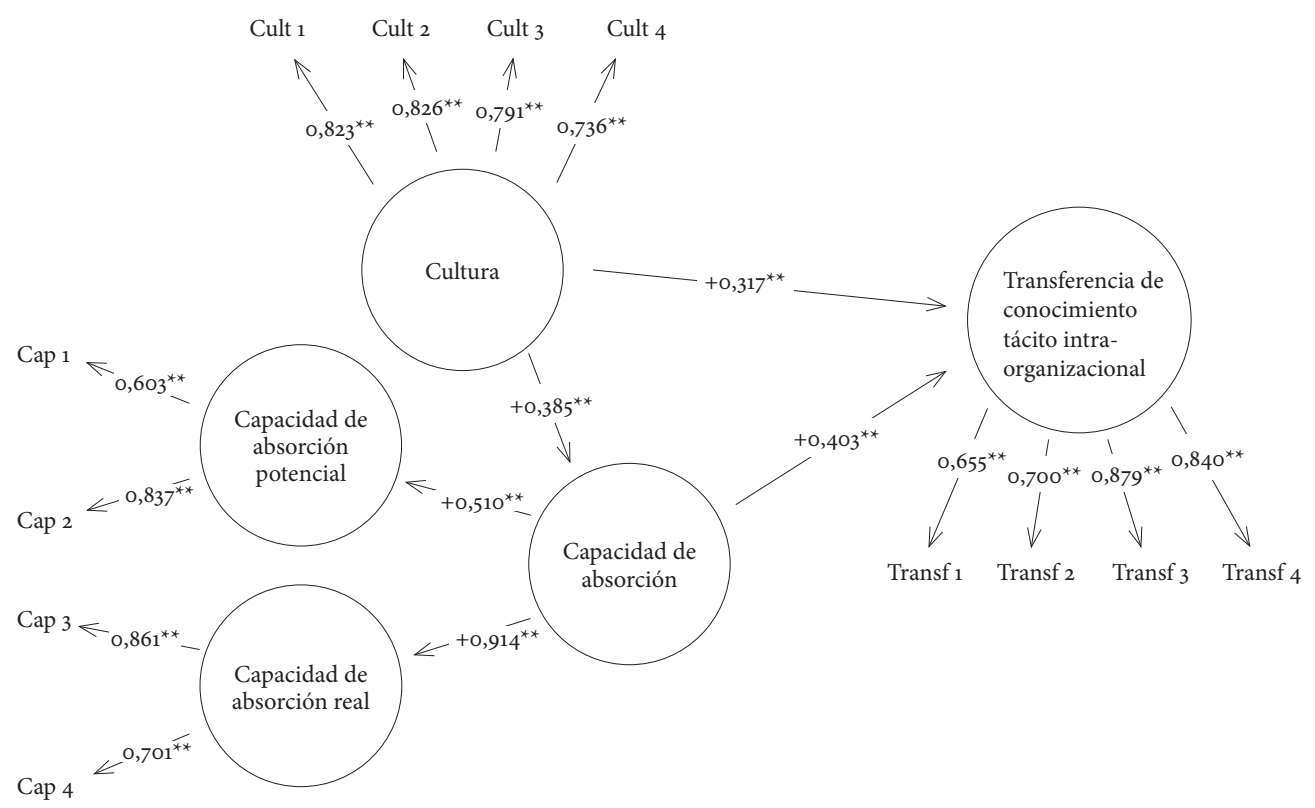

Nota. ${ }^{*} \mathrm{p}<0,05,{ }^{* *} \mathrm{p}<0,01$.

Fuente: Elaboración propia.

Tabla 5. Cálculo de los efectos totales sobre las variables endógenas del modelo

\begin{tabular}{lcccc}
\hline $\begin{array}{l}\text { Influencia de la cultura orga- } \\
\text { nizacional sobre: }\end{array}$ & $\begin{array}{l}\text { Efectos } \\
\text { directos }\end{array}$ & Efectos indirectos & Efectos totales \\
\hline $\begin{array}{l}\text { Transferencia de Conocimiento } \\
\text { tácito Intra-organizacional }\end{array}$ & $0,317^{* *}$ & Cult $\rightarrow$ CapAbs $\rightarrow$ Transf & $0,155^{* *}$ & $0,472^{* *}$ \\
Capacidad de absorción & $0,385^{* *}$ & NA & $0,385^{* *}$ \\
\hline
\end{tabular}

Nota. ${ }^{*} \mathrm{p}<0,05 ;{ }^{*} \mathrm{p}<0,01 ; \mathrm{NA}=$ No aplica.

Fuente: Elaboración propia.

cultura también impacte la estrategia de la organización, ya que la evidencia empírica indica que las entidades que tengan en mente generar o apuntalar su ventaja competitiva sostenible, pueden considerar como alternativa viable a la transferencia de conocimiento tácito interna (Máynez, 2011), y la literatura indica que esta actividad intraorganizacional se valora como un elemento crítico en la implementación de cualquier tipo de estrategia (Bloodgood \& Morrow, 2003). Además, dado que la cultura afecta positivamente a la transferencia interna de conocimiento tácito (Lucas \& Ogilvie, 2006), se convierte en un aspecto clave para facilitar los esfuerzos de gestión del conocimiento (Yoo \& Torrey, 2002), representando un elemento de vital importancia, tanto en términos del tipo de conocimiento, como del método utilizado para compartirlo (Jonsson \& Kalling, 2007).

Por otro lado, los resultados obtenidos en la realidad organizacional confirman que la capacidad de absorción individual 
afecta de forma positiva y significativa a la transferencia de conocimiento tácito dentro de la organización, ya que el aprendizaje individual resulta mayor cuando el nuevo conocimiento se relaciona con el ya existente (Lane \& Lubatkin, 1998); además a partir de éste, se faculta al individuo para que sea capaz de encontrarle sentido, asimilarlo, transformarlo y utilizarlo (Cohen \& Levinthal, 1990; Kim, 1999; Zahra \& George, 2002).

Por otra parte, los antecedentes muestran una carencia de evidencia empírica respecto de la influencia de la cultura sobre la capacidad de absorción (Murovec \& Prodan, 2009), habiéndose analizado de forma exploratoria algunas de las relaciones entre estas variables (Khoja \& Maranville, 2010). Para incrementar el conocimiento relativo, esta investigación se abordó desde una perspectiva confirmatoria, y los resultados permiten afirmar que la cultura incide de forma positiva y significativa sobre la capacidad de absorción y que ésta última modera la influencia de la primera sobre la transferencia de conocimiento tácito intra-organizacional, en el contexto industrial estudiado.

Para seguir avanzando en el conocimiento de estas variables y dado que la capacidad de absorción de la firma depende de la capacidad de absorción de sus trabajadores (Cohen \& Levinthal, 1990), resulta relevante investigar la forma en la que éstas inciden sobre los procesos de innovación. $\mathrm{Al}$ respecto, la literatura indica que cuando un departamento tenga acceso a cierto conocimiento novedoso, no garantiza que se afiancen sus capacidades de innovación y desempeño, sobre todo si se carece de capacidad suficiente para absorberlo (Tsai, 2001), ya que si el receptor no es capaz de aprender exitosamente el conocimiento que se le comparte y de asimilar el contenido valioso del mismo, es menos posible que lo aplique para resolver sus propios problemas (Ho \& Gao, 2005).

Además, la evidencia señala que la interacción entre los empleados con estructuras de conocimiento diferentes incrementará la capacidad de la empresa para hacer nuevas relaciones y asociaciones, logrando que la innovación vaya más allá de lo que un sujeto en lo individual pueda lograr (Cohen \& Levinthal, 1990), y en el mismo sentido existen planteamientos que afirman que las organizaciones que promueven que se compartan los conocimientos, fomentan que las áreas afiancen su capacidad de absorción (Khoja \& Maranville, 2010).

Un tema central en la administración estratégica es la propuesta que las asimetrías de conocimiento entre las organizaciones generan diferencias de desempeño entre las mismas (Thomas, Watts \& Henderson, 2001). Los cambios y presiones de una economía global, rápidamente cambiante y basada en la información, han provocado que el conocimiento sea un activo vital para las organizaciones (Herschel \& Nemati, 2000), ya que faculta a las personas y a las empresas a resolver problemas y a ser competitivas (Evanschitzky et al., 2007). Las habilidades, las competencias y el conocimiento, son recursos esenciales en la economía del conocimiento, y muchas organizaciones han tomado conciencia de que la administración de éstos es una actividad clave para sobrevivir y ser rentables (Hafeez \& Abdelmeguid, 2003). Compartir el conocimiento tiene la posibilidad de mejorar el desempeño de las empresas y acrecentar la ventaja competitiva (Hsu, 2008). Desde el punto de vista estratégico, el conocimiento tácito es un recurso muy utilizado por parte de las empresas que, como estrategia, reconfiguran sus recursos organizacionales existentes (Bloodgood \& Morrow, 2003). Además, activos intangibles como el conocimiento técnico, el diseño del 
producto, el conocimiento del cliente, la presentación comercial, la creatividad y la innovación, se fundamentan en el conocimiento (Herschel \& Nemati, 200o).

La transferencia de conocimiento es una actividad mediadora que faculta el logro de los objetivos organizacionales ya que da soporte a la innovación y, por tanto, a que se alcancen ventajas competitivas sostenibles, sobre todo si se trata de la transmisión de conocimiento tácito, caracterizado por sus atributos de valioso, inimitable, único e insustituible. Por lo anterior, se recomienda estudiar las relaciones entre la transferencia de conocimiento tácito y la innovación. Asimismo, es recomendable que en estudios posteriores se repliquen las relaciones de los constructos estudiados bajo muestras probabilísticas, ampliando los contextos y diversificando las características de los sujetos de estudio, lo cual incidirá positivamente sobre la validez externa del modelo.

\section{Referencias bibliográficas}

Alavi, M., Kayworth, T.R. \& Leidner, D.E. (2005). An Empirical Examination of the Influence of Organizational Culture on Knowledge Management Practices. Journal of Management Information Systems, 22(3), 191-224.

Anderson, J.C. \& Gerbing, D.W. (1988). Structural Equation Modeling in Practice: A Review and Recommended Two-Step Approach. Psychological Bulletin, 103(3), 411-423.

Argote, L. \& Ingram, P. (2000). Knowledge Transfer: A Basis for Competitive Advantage in Firms. Organizational behavior \& human decision processes, 82(1), 150-169.

Bagozzi, R.P. \& Yi, Y. (1988). On the Evaluation of Structural Equation Models.
Journal of the Academy of Marketing Science, 16(1), 74-94.

Bloodgood, J. \& Morrow, J. (2003). Strategic organizational change: exploring the roles of environmental structure, internal conscious awareness and knowledge. Journal of Management Studies, 4o(7), 1761-1782. Cameron, K.S. \& Quinn, R.E. (2006). Diagnosis and changing organizational culture: based on the competing values framework (revised ed.). San Francisco, CA: JosseyBass.

Chan, K.Y., Pretorius, M.W. \& Oerlemans, L.A.G. (2012). A relational view of knowledge transfer effectiveness in small new technology-based firms: An empirical analysis of a South African case. African Journal of Business Management, 6(11), 3930-3940.

Cohen, W.M. \& Levinthal, D.A. (1990). Absorptive Capacity: A New Learning Perspective on Learning and Innovation. Administrative Science Quarterly, 35(1), 128-152.

Collins, J. \& Hitt, M. (2006). Leveraging tacit knowledge in alliances: The importance of using relational capabilities to build and leverage relational capital. Journal of Engineering \& Technology Management Jet- $M$, 23(3), 147-167.

Collins, J. \& Smith, K.G. (2006). Knowledge exchange and combination: the role of human resource practices in the performance of high-technology firms. Academy of Management Review, 49(3), 544-560.

Davenport, T.H. \& Prusak, L. (2000). Working Knowledge: How Organizations Manage What They Know. Boston, MA: Harvard Business School Press.

De Long, D.W. \& Fahey, L. (2000). Diagnosing cultural barriers to knowledge management. Academy of Management Executive, 14(4), 113-127. 
Deng, X., Doll, W.J. \& Cao, M. (2008). Exploring the absorptive capacity to innovation/productivity link for individual engineers engaged in IT enabled work. Information Management, 45(2), 75-87.

Dixon, N. (2002). The neglected receiver of knowledge sharing. Ivey Business Journal, 66(4), 35-40.

Donate, M.J. \& Guadamillas, F. (2010). The Effect of Organizational Culture on Knowledge Management Practices and Innovation. Knowledge \& Process Management, 17(2), 82-94.

Evanschitzky, H., Ahlert, D., Blaich, G. \& Kenning, P. (2007). Knowledge management in knowledge-intensive service networks. A strategic management approach. Management Decision, 45(2), 265-283.

Flatten, T.C., Engelen, A., Zahra, S.A. \& Brettel, M. (2011). A measure of absorptive capacity: Scale development and validation. European Management Journal, 29(2), 98-116.

Fornell, C. \& Larcker, D.F. (1981). Evaluating structural equation models with unobservable variables and measurement error. Journal of Marketing Research, 18(1), 39-50.

Gold, A.H., Malhotra, A. \& Segars, A.H. (2001). Knowledge Management: An Organizational Capabilities Perspective. Journal of Management Information Systems, 18(1), 185-214.

Grant, R. (1996). Toward a knowledgebased theory of the firm. Strategic Management Journal, 17 (Winter Special Issue), 109-122.

Hafeez, K. \& Abdelmeguid, H. (2003). Dynamics of human resource and knowledge management. Journal of Operational Research Society, 54(2), 153-164.

Hair, J., Bush, R. \& Ortinau, D. (2007). Investigación de Mercados en un ambiente de información cambiante (2a. ed.). México: McGraw-Hill.

Hair, J., Jr., Anderson, R.E., Tatham, R.L. \& Black, W.C. (1999). Análisis Multivariante ( 5 a ed.). Madrid: Prentice Hall Iberia.

Hall, R. (1992). The Strategic Analysis of Intangible Resources. Strategic Management Journal, 13(2), 135-144.

Harrington, S.J. \& Guimaraes, T. (2005). Corporate culture, absorptive capacity and IT success. Information and Organization, 15(1), 39-63.

Hasnain, S.S. \& Jasimuddin, S.M. (2012). Barriers to Knowledge Transfer: Empirical Evidence from the NGO (NonGovernmental Organizations) - Sector in Bangladesh. World Journal of Social Sciences, 2(2), 135-150.

Herschel, R.T. \& Nemati, D.R. (200o). Chief knowledge officer: critical success factors for knowledge management. Information Strategy: The Executive's Journal, 16(4), 37-45.

Ho, S. \& Gao, S. (2005). Attitude towards knowledge sharing behavior. Journal of Computer Information Systems, 46(2), 45-51.

Hsu, I.C. (2008). Knowledge sharing practices as a facilitating factor for improving organizational performance through human capital: A preliminary test. Expert Systems with applications, 35(3), 13161326.

Hu, L.T. \& Bentler, P.M. (1999). Cutoff Criteria for Fit Indexes in Covariance Structure Analysis: Conventional Criteria Versus New Alternatives. Structural Equation Modeling, 6(1), 1-55.

Inkpen, A.C. \& Pien, W. (2006). An Examination of Collaboration and Knowledge Transfer: China-Singapore Suzhou Industrial Park. Journal of Management Studies, 43(4), 779-811. 
Itami, H. \& Roehl, T.W. (1987). Mobilizing Invisible Assets. Cambridge, MA: Harvard University Press.

Jonsson, A. \& Kalling, T. (2007). Challenges to knowledge sharing across national and intra-organizational boundaries: case studies of IKEA and SCA Packaging. Knowledge Management Research \& Practice, 5(3), 161-172.

Khoja, F. \& Maranville, S. (2010). How Do Firms Nurture Absorptive Capacity? Journal of Managerial Issues, 22(2), 262-278.

Kim, L. (1999). Building technological capability for industrialization: analytical frameworks and Korea's experience. Industrial \& Corporate Change, 8(1), 111-136.

Knoppen, D., Sáenz, M.J. \& Johnston, D.A. (2011). Innovations in a relational context: Mechanisms to connect learning processes of absorptive capacity. Management Learning, 42(4), 419-438.

Kogut, B. \& Zander, U. (1992). Knowledge of the firm, combinative capabilities and replication of technology. Organization Science, 3(3), 383-397.

Kostova, T. (1999). Transnational Transfer of Strategic Organizational Practices: A Contextual Perspective. Academy of Management Review, 24(2), 308-324.

Lane, P.J. \& Lubatkin, M. (1998). Relative absorptive capacity and interorganizational learning. Strategic Management Journal, 19(5), 461-477.

Lichtenthaler, U. (2009). Absorptive capacity, environmental turbulence, and the complementarity of organizational learning processes. Academy of Management Journal, 52(4), 822-846.

Likert, R. (1932). A technique for the measurement of attitudes. Archives of Psychology, 22(140), 1-55.

Lin, C., Tan, B. \& Chang, S. (2008). An exploratory model of knowledge flow barriers within healthcare organizations. Information \& Management, 45(5), 331339.

Liyanage, C., Elhag, T., Ballal, T. \& Li, Q. (2009). Knowledge communication and translation - a knowledge transfer model. Journal of Knowledge management, 13(3), 118-131.

Lucas, L.M. \& Ogilvie, D. (2006). Things are not always what they seem: How reputations, culture, and incentives influence knowledge transfer. The Learning Organization, 13(1), 7-24.

Máynez, A.I. (2011). La Transferencia de Conocimiento Organizacional como Fuente de Ventaja Competitiva Sostenible: Modelo Integrador de Factores y Estrategias. Puebla, México: Universidad Popular Autónoma del Estado de Puebla.

Miller, D.J., Fern, M.J. \& Cardinal, L.B. (2007). The use of knowledge for technological innovation within diversified firms. Academy of Management Journal, 50(2), 308-326.

Minbaeva, D.B. (2007). Knowledge transfer in multinational corporations. Management International Review, 47(4), 567-593. Murovec, N. \& Prodan, I. (2009). Absorptive capacity, its determinants, and influence on innovation output: Cross-cultural validation of the structural model. Technovation, 29(12), 859-872.

Nunnally, J. \& Bernstein, I. (1994). Psychometric theory (3a ed.). New York, NY: McGraw-Hill.

O'Reilly, C.A., III, Chatman, J. \& Caldwell, D.F. (1991). People and Organizational Culture: A Profile Comparison to Assessing Person-Organization Fit. The Academy of Management Review, 34(3), 487-516.

Peansupap, V. \& Walker, D.H.T. (2009). Exploratory factors influencing design practice learning within a Thai context. 
Engineering, Construction and Architectural Management, 16(3), 238-253.

Penrose, E. (1962). Teoría del crecimiento de la empresa (F.V. Parache, Trans.). Madrid: Aguilar.

Perrin, A., Rolland, N. \& Stanley, T. (2007). Achieving best practices transfer across countries. Journal of Knowledge Management, 11(3), 156-166.

Reagans, R. \& McEvily, B. (2003). Network Structure and Knowledge Transfer: The Effects of Cohesion and Range. Administrative Science Quarterly, 48(2), 240-267.

Reed, R. \& De Fillippi, R. (1990). Causal ambiguity, barriers to imitation, and sustainable competitive advantage. Academy of Management Review, 15(1), 88-102.

Riege, A. (2005). Three-dozen knowledgesharing barriers managers must consider. Journal of Knowledge Management, 9(3), 18-35.

Santoro, M.D. \& Bierly, P. (2006). Facilitators of knowledge transfer in UniversityIndustry collaborations: a knowledgebased perspective. IEEE Transactions in Engineering Management, 53(4), 495-507. Satorra, A. \& Bentler, P.M. (1994). Corrections to test statistics and standard errors in covariance structure analysis. En A. von Eye \& C.C. Clogg (Eds.), Latent variable analysis: Applications for developmental research. Thousand Oaks, CA: Sage.

Schein, E.H. (2004). Organizational Culture and Leadership (3a ed.). San Francisco, CA: Jossey-Bass.

Seidler-de Alwis, R. \& Hartmann, E. (2008). The use of tacit knowledge within innovative companies: knowledge management in innovative enterprises. Journal of knowledge Management, 12(1), 133-147.

Simonen, J. \& McCann, P. (2010). Knowledge transfers and innovation: The role of labour markets and R\&D co-operation between agents and institutions. Papers in Regional Science, 89(2), 295-309.

Simonin, B. (2004). An Empirical Investigation of the Process of Knowledge Transfer in International Strategic Alliances. Journal of International Business Studies, 35(5), 407-427.

Swart, J., van den Hooff, B. \& van Baalen, P. (2011). Connecting Worlds. Management Learning, 42(4), 371-377.

Szulanski, G. (1996). Exploring Internal Stickiness: Impediments to the Transfer of Best Practice Within the Firm. Strategic Management Journal, 17(Special Issue: Knowledge and the Firm), 27-43.

Tallman, S., Jenkins, M., Henry, N. \& Pinch, S. (2004). Knowledge, clusters, and competitive advantage. Academy of Management Review, 29(2), 258-271.

Thomas, J., Watts, S. \& Henderson, J. (2001). Understanding "strategic learning": linking organizational learning, knowledge management, and sense making. Organization Science, 12(3), 331-345.

Tsai, W. (2001). Knowledge Transfer in intraorganizational networks: Effects of network position and absorptive capacity on business unit innovation and performance. The Academy of Management Journal, 44(5), 996-1004.

Tsoukas, H. \& Vladimirou, E. (2001). What is organizational knowledge? Journal of Management Studies, 38(7), 973-993.

Wijk, R., Jansen, J.J.P. \& Lyles, M.A. (2008). Inter- and Intra-Organizational Knowledge Transfer: A Meta-Analytic Review and Assessment of its Antecedents and Consequences. Journal of Management Studies, 45(4), 830-853.

Wong, K.Y. \& Aspinwall, E. (2005). An empirical study of the important factors for knowledge-management adoption in the SME sector. Journal of Knowledge Management, 9(3), 64-82. 
Xinhua, B. \& Cuiling, Y. (2008). Absorptive Capacity of Information Technology and Its Conceptual Model. Tsinghua Science and Technology 13(3), 337-343.

Yoo, Y. \& Torrey, B. (2002). National Culture and Knowledge Management in a Global Learning Organization: A Case Study. In C. W. Choo \& N. Bontis (Eds.), The Strategic Management of Intellectual Capital and Organizational Knowledge (pp. 421-435). New York, NY: Oxford University Press.

Zahra, S.A. \& George, G. (2002). Absorptive Capacity: a review, reconceptualization, and extension. Academy of Management Review, 27(2), 185-203.

Zhao, Z.J. \& Anand, J. (2009). A multilevel perspective on knowledge transfer: evidence from the Chinese automotive industry. Strategic Management Journal, 3o(9), 959-983. 